\title{
Towards Communication UAV-Based: Improving Throughput By Optimum Trajectory And Power Allocation
}

\section{Sedighe Nasrollahi}

Shahrood University of Technology

Seyed Masoud Mirrezaei ( $\nabla$ sm_mirrezai@yahoo.com )

Shahrood University of Technology https://orcid.org/0000-0001-8493-2072

\section{Research}

Keywords: UAVs, Relay node, Optimization, Power allocation, UAV trajectory

Posted Date: July 23rd, 2021

DOl: https://doi.org/10.21203/rs.3.rs-692648/v1

License: (c) (i) This work is licensed under a Creative Commons Attribution 4.0 International License.

Read Full License

Version of Record: A version of this preprint was published at EURASIP Journal on Wireless Communications and Networking on January 21st, 2022. See the published version at https://doi.org/10.1186/s13638-022-02087-6. 


\title{
RESEARCH
}

\section{Towards Communication UAV-based: Improving Throughput by optimum Trajectory and Power Allocation}

\author{
Sedighe Nasrollahi and Seyed Masoud Mirrezaei*
}

\author{
${ }^{*}$ Correspondence: \\ sm.mirrezaei@shahroodut.ac.ir \\ Faculty of Electrical Engineering, \\ Shahrood University of \\ Technology, Shahrood, Iran \\ Full list of author information is \\ available at the end of the article
}

\begin{abstract}
It is predicted that the use of unmanned aerial vehicles (UAVs) in communication systems will be more extensive in future generations of wireless

telecommunication networks, due to their facilitating advantages. In this paper, a UAV-based wireless communication system is considered in which a UAV is employed as a relay to connect two ground users. These two disconnected users make a communication pair. Our aim is to maximize the minimum achievable information rate for the communication link between the transmitter and receiver, by cooperatively optimizing UAV trajectory and transmitter and source power allocation. Motivated by the above, we formulate the optimization problem. The solving process is complicated because of the non-convexity of the formulated problem. To overcome this difficulty, we convert the main problem to some sub-problems by fixing some constraints and solve them with iterative algorithms such as successive convex optimization and reach the solution for the main problem. Simulation results show the capability of the proposed algorithm.
\end{abstract}

Keywords: UAVs; Relay node; Optimization; Power allocation; UAV trajectory

\section{Introduction}

By increasing mobile users in 5G and also the expansion of internet of things (IoT) applications in future communication systems, communication networks face a high amount of data traffic that is out of available terrestrial base station's capacity. To overcome this challenge and coverage improvement of communication networks, relaying is an effective technique [1,2]. As most of the communication systems are wired, conventional relaying systems are based on static relaying. Due to cost reduction, device miniaturization, and other advantages, UAVs can be employed as relays in wireless networks. In addition to relaying, UAVs can be used in other applications such as power transfer, data collection, information broadcasting, cargo delivery, traffic monitoring, and emergency situations for example disaster management. These applications benefit from UAV's mobility and on-demand deployment and also the line-of-sight channel between UAV and considered ground transmitter or receiver [3]. According to [4] and 3GPP [5], for the moderate altitude of UAV for example more than 50 meters, the LOS probability is more than 90 percent. So, the channel may be less affected by shadowing, fading, and multipath propagation. Since the horizontal placement of the UAV can be changed, the channel between the UAV and ground nodes has variation. Motivated by this, UAV can get closer to the objective area and achieve a better communication channel with 
more gain. As discussed in [6,7]another parameter of a UAV's placement is its altitude that influences the coverage probability and system sum rate. UAVs or drones are categorized into two types including fixed-wing and rotary-wing UAVs. The difference between them is that the speed of fixed-wing UAVs is higher and they have weighty cargo, but they must maintain uninterrupted ahead movement to hover overhead, and thus are not appropriate for stationary applications. Unlike this, rotary-wing UAVs, although having limited mobility and payload, are able to move in any direction and stay motionless in the air. Hence, the choice of UAV type entirely depends on the applications [8]. Based on practical applications, various research endeavors have been dedicated to UAV-aided wireless networks in last decade. In [9], a UAV-enabled data collection system was studied where a UAV is sent out to gather a given amount of data from ground terminals (GT) at known fixed locations. In this paper, the propulsion energy of the UAV was considered to be controlled due to its finite onboard energy. UAV-enabled wireless power transfer (WPT) system with trajectory design and energy optimization was investigated in [10]. This research was extended to simultaneous wireless information and power transfer (SWIPT) in cooperative communication systems in [11]. In this paper, the UAV's transmission ability is powered entirely by radio frequency signal, transmitted from the source via time-sharing mechanism. Energy minimization is another important factor in designing UAV-enabled systems. Though initial attempts for designing energy-efficient UAV communication was appropriated to fixed-wing UAV enabled communication system by maximizing its energy efficiency in bits/Joule [12], authors in [13] investigated energy-efficient communication design for rotary-wing UAVs in a multi-user situation. Another application of UAVs is its role in the field of Internet of Things in which the device's transmit power is poor so they are not able to communicate over a long area. In such a case, the UAV is a mean to collect the IoT data from the transmitter device and send it to its corresponding receiver [14]. In natural disasters, IoT coverage will be extremely affected, due to the destruction of communications infrastructure. In such cases, having an emergency communication network can be a crucial factor in getting rid of the status quo. UAVs can be applied to implement public safety scenarios to support disaster alleviation measures [15-17]. Co-existence between the UAV and device-to-device (D2D) communication network is another subject that has been addressed in $[18,19]$. In these researches, the deployment of UAV as a flying base station for a desired geographical area is analyzed and the most focus is on the coverage probability. Energy harvesting is a new technology for increasing network lifetime. Unlike other devices, an energy harvesting device can receive energy from renewable sources in the environment and provide continuous power supply to wireless devices. In [20], the resource allocation problem for energy harvesting powered D2D communication underlaying UAV-assisted Networks was studied. The security issue of UAV networks is critical to have a protected communication. The authors in [21] considered boosting physical layer security by using mobile relaying system, in which a UAV acts as a mobile relay and flexibly regulates its location in order to enhance the desired wireless communication security. In [22-24], an optimization problem has been formulated to maximize the minimum average secrecy rate over all receivers by jointly optimizing UAV trajectory and transmit 
power. Considering UAV as a mobile relay is an extensive issue of researches mentioned in $[25,26]$. In [27-30], the scenario of multiple UAVs deployed as areal base stations and relays, respectively, was studied.

In this paper, we consider one disconnected communication pair and a UAV ministering as a mobile relay to establish connectivity between the source and destination node. Our goal is to maximize the minimum information rate for our communication pair by cooperatively optimizing UAV trajectory and source and relay transmission power. The formulated optimization problem is difficult to solve due to its non-convexity. To make the problem tractable, we partition the main problem into two sub-problems and solve them by successive convex optimization technique. Then an overall algorithm is produced to solve two sub-problems in an alternate manner. The rest of this paper is organized as follows. In section 2our desired UAV-relayed system model and the problem formulation are introduced. Section 3 proposes the iterative algorithm based on SCO. In section 4, we present the simulation results to verify the effectiveness of the proposed algorithm. Finally, we conclude this paper in section 5 .

\section{System Model and Problem Formulation}

Consider the scenario that in a disaster (or any reason that destroys the communication infrastructure) area, two disconnected ground nodes that are at a distance of $\mathrm{L}$ meters apart, cannot communicate with each other due to long distance or severe blockage. Thus, a UAV relay is deployed to establish communication link between source and destination defined by A and B, respectively. We assume that A and $\mathrm{B}$ are located on the ground with known fixed locations and the flight height of the UAV from the ground is constant and equal to $\mathrm{H}$ in a period of $\mathrm{T}$ seconds. The horizontal coordinates of A and B and UAV are $(0,0,0),(L, 0,0)$ and $(x(t), y(t), H)$, respectively. The analytical model of such a system is shown in Fig.1.

Note that we assume this scenario for real-time applications such as building interconnection in emergency situations; therefore, the amplify-and-forward (AF) strategy is more suitable than decode-and-forward (DF) due to less complexity. For ease of analysis, the time horizon $\mathrm{T}$ is discretized into $\mathrm{N}$ equal time slots. The parameter $\mathrm{N}$ should be chosen large enough or in other words the elemental slot length be small enough, so that the position of UAV is approximately constant at any time slot. Thus, the trajectory of UAV over $\mathrm{T}$ can be rewritten as $(x[n], y[n]), \forall n=1, \cdots, N$. But increasing $\mathrm{N}$ will bring more computational complexity. In fact, while choosing the value of $\mathrm{N}$, we should consider a tradeoff between the accuracy and complexity [27]. Motivated by AF protocol, the UAV transmits data to B, as soon as received it from A. We partition each time slot into 2 hops. Sending data from source to the UAV happens in the first hop. So, the received signal at $\mathrm{UAV}$ in the nth time slot can be expressed as

$$
Y_{U}[n]=\sqrt{P_{A}[n] h_{A U}[n]} X[n]+Z_{1}[n]
$$

Where $P_{A}[n]$ is the transmission power of A. $X_{A}[n]$ is the transmitted signal to $\mathrm{UAV}$ and $Z_{1}[n] \sim N\left(0, \sigma^{2}\right)$ is the power of additive white Gaussian noise (AWGN) observed at UAV. $h_{A} U[n]$ represents the LOS channel between UAV and source. 
Figure 1 The analytical model of the UAV-relayed wireless system

Considering the free-space pass loss model and ignoring the Doppler effect caused by UAV mobility, the channel power gain from source to UAV can be expressed as

$$
h_{A U}[n]=\alpha_{0} d_{A U}^{-2}[n]=\frac{\alpha_{0}}{x^{2}[n]+y^{2}[n]+H^{2}}
$$

where $\alpha_{0}$ illustrates the reference channel power at the distance $d_{0}=1 \mathrm{~m}$. As we see the channel power depends only on the UAV-user distance. For the second hop, the UAV scales the received signal and broadcasts it to the destination with gain $G[n]$ as follow

$$
G[n]=\sqrt{\frac{P_{U}[n]}{P_{A}[n] h_{A U}[n]+\sigma^{2}}}
$$

where $P_{U}[n]$ is the transmission power of UAV. Thus the signal received at B can be written as follows

$$
Y_{B}[n]=G[n] \sqrt{h_{U B}[n]} Y_{U}[n]+Z_{2}[n]
$$

where $Z_{2}[n] \sim N\left(0, \sigma^{2}\right)$ is the power of additive white Gaussian noise at destination. The following equation shows the channel gain of UAV-B link.

$$
h_{U B}[n]=\alpha_{0} d_{U B}^{-2}[n]=\frac{\alpha_{0}}{(x[n]-L)^{2}+y^{2}[n]+H^{2}}
$$

In the above expressions, $d_{A U}[n]$ and $d_{U B}[n]$ are the link distance between source and UAV, and UAV and destination at time slotn. Considering (3) and (4), the 
corresponding signal-to-noise ratio (SNR) at the destination can be written as

$$
\gamma[n]=\frac{P_{A}[n] P_{U}[n] h_{A U}[n] h_{U B}[n]}{\left(P_{A}[n] h_{A U}[n]+P_{U}[n] h_{U B}[n]+\sigma^{2}\right) \sigma^{2}}
$$

The accessible information rate for the source to destination link at nth time slot can be expressed as

$$
R[n]=\frac{1}{2} \log _{2}(1+\gamma[n]), n=1, \cdots, N
$$

The goal is maximizing the minimum of this rate by optimizing both source/UAV power allocation and UAV trajectory. By defining $P \triangleq\left(P_{A}[n], P_{U}[n]\right)$ and $W \triangleq$ $(x[n], y[n])$, the optimization problem can be formulated as

$$
\begin{aligned}
& (P 1): \max _{P, W} \min R[n], n=1, \cdots, N . \\
& \text { s.t. } \sum_{n=1}^{N} P_{A}[n] \leq N \overline{P_{A}}, \sum_{n=1}^{N} P_{U}[n] \leq N \overline{P_{U}} \\
& P_{A}[n] \geq 0, P_{U}[n] \geq 0, n=1, \cdots, N . \\
& (x[n+1]-x[n])^{2}+(y[n+1]-y[n])^{2} \leq\left(\frac{V T}{N}\right)^{2}, n=1,2, \cdots, N-1
\end{aligned}
$$

where $\overline{P_{A}}$ and $\overline{P_{U}}$ are the average maximum transmission power of source $\mathrm{A}$ and UAV. By defining $\mathrm{V}$ as the maximum permitted flying speed of UAV, $\frac{V T}{N}$ represents the maximum horizontal distance the UAV can fly in each time slot. Constraint (8d) implies that the distance that the UAV travel in one time slot shouldn't exceed its maximum value. The max-min optimization problem is non-convex because the logarithmic objective function is not convex. In our proposed method we suggest an iterative algorithm to solve this problem.

\section{Proposed Method}

As we say the main optimization problem is non-convex and incurable to solve. In this part, we introduce two sub-problems and develop an iterative algorithm to solve them alternately to achieve the solution for the main problem. First, we solve the optimization problem with fixed UAV trajectory and obtain the source/relay power allocation and then repeat with fixed power allocation to obtain the optimal trajectory. Finally, the overall algorithm is proposed.

\subsection{Power Optimization with Fixed UAV Trajectory}

By assuming the UAV trajectory fixed, the constraints reduce to ones that are only on the power. Also, the varying channel is known due to the pre-determined trajectory. So, the main problem can be written as the following form

$$
(P 1.1): \max _{P} \min R[n], n=1, \cdots, N
$$




$$
\text { s.t. (11b) and (11c) }
$$

The objective function doesn't change. So, the problem (P1.1) is still non-convex. To cope with this non-convexity, we utilize iterative approximation helping from successive convex optimization techniques. As mentioned in [31], any convex function is lower-bounded by its first-order Taylor expansion. Motivated by this, we maximize the lower bound of our objective function by optimizing the source and UAV's power in each iteration. We can write the Taylor expansion of the transmission rate at $\frac{1}{\gamma[n]}$ as follow

$$
\begin{aligned}
& f(x) \geq f\left(x_{0}\right)+f\left(x_{0}\right)\left(x-x_{0}\right), \forall x \rightarrow \\
& R_{l+1}[n] \geq R_{l}[n]-\frac{\gamma_{l}^{2} \log _{2} e}{2\left(\gamma_{l}[n]+1\right)}\left(\frac{1}{\gamma_{l+1}[n]}-\frac{1}{\gamma_{l}[n]}\right)=R_{l b, l+1}, n=1, \cdots, N
\end{aligned}
$$

In the above expressions, $l$ and $l+1$ indexes introduce $l$ th and $(l+1)$ th iterations. From the equation of SNR calculation, it is obvious that $\gamma[n]$ is not convex with respect to $P_{A}[n]$ and $P_{U}[n]$. So, we can say that $\frac{1}{\gamma[n]}$ is a convex function of $P_{A}[n]$ and $P_{U}[n]$. Therefore, we can rewrite the above problem as

$$
\begin{aligned}
& (P 1.2): \max _{P} \min R_{l b, l+1}[n], n=1, \cdots, N . \\
& \text { s.t. } \sum_{n=1}^{N} P_{A, l+1}[n] \leq N \overline{P_{A}}, \sum_{n=1}^{N} P_{U, l+1}[n] \leq N \overline{P_{U}} \\
& P_{A, l+1}[n] \geq 0, \quad P_{U, l+1}[n] \geq 0, n=1, \cdots, N .
\end{aligned}
$$

(P1.2) is a convex version of (P1.1) and can be efficiently solved by existing standard convex optimization tools such as YALMIP. The optimal solution of (P1.1) is also lower-bounded by the solution of (P1.2).

\subsection{Trajectory Optimization with Fixed Power}

In this part, the trajectory optimization problem is solved for any desired source or UAV power. This problem can be summarized as

$$
\begin{aligned}
& (P 1.3): \max _{W} \min R[n], n=1, \cdots, N \\
& \text { s.t. (8d) }
\end{aligned}
$$

Again, we face a non-convex optimization problem because of the non-convex objective function and should utilize successive convex optimization method to find its optimal solution efficiently. We define $\left\{x_{l}[n], y_{l}[n]\right\}$ and $\left\{x_{l+1}[n], y_{l+1}[n]\right\}$, the final location of UAV after $l$ th and $(l+1)$ th iteration. Since the non-convexity of the objective function is concerning $\{x[n], y[n]\}$, we present two new variables named $s_{1}[n]=\frac{1}{h_{A U}[n]}$ and $s_{2}[n]=\frac{1}{h_{U B}[n]} \cdot \gamma[n]$ is convex with respect to $s_{1}[n]$ and $s_{2}[n]$ and 
first-order Taylor expansion can be used to approximate it. Firstly, we rewrite the $\gamma[n]$ according to new variables as below

$$
\gamma[n]=\frac{P_{A}[n] P_{U}[n]}{\left(P_{A}[n] s_{2}[n]+P_{U}[n] s_{1}[n]+\sigma^{2} s_{1}[n] s_{2}[n]\right) \sigma^{2}}
$$

Using Taylor expansion, we have

$$
\gamma_{l+1}[n] \geq \gamma_{l}[n]-C_{l}[n]\left(s_{1, l+1}[n]-s_{1, l}[n]\right)-D_{l}[n]\left(s_{2, l+1}[n]-s_{2, l}[n]\right)=\gamma_{l b, l+1}[n]
$$

where $C_{l}[n]$ and $D_{l}[n]$ are the gradients of the $\gamma[n]$ at new variables which can be calculated by

$$
\begin{aligned}
& C_{l}[n]=\frac{P_{A}[n] P_{U}[n]\left(P_{U}[n] \sigma^{2}+\sigma^{4} s_{2, l}[n]\right)}{\left(P_{A}[n] s_{2}[n]+P_{U}[n] s_{1}[n]+\sigma^{2} s_{1}[n] s_{2}[n]\right)^{2} \sigma^{4}} \\
& D_{l}[n]=\frac{P_{A}[n] P_{U}[n]\left(P_{A}[n] \sigma^{2}+\sigma^{4} s_{1, l}[n]\right)}{\left(P_{A}[n] s_{2}[n]+P_{U}[n] s_{1}[n]+\sigma^{2} s_{1}[n] s_{2}[n]\right)^{2} \sigma^{4}}
\end{aligned}
$$

From $\gamma_{l b}[n]$ we have the lower bound of transmission rate as

$$
R_{l b, l+1}[n]=\frac{1}{2} \log _{2} 1+\gamma_{l b, l+1}[n]
$$

Now the convex optimization problem for trajectory optimization for given source and trajectory optimization scenario can be summarized as

$$
\begin{aligned}
& \qquad(P 1.4): \max _{W} \min R_{l b, l+1}[n], n=1, \cdots, N \\
& \text { s.t. }\left(x_{l+1}[n+1]-x_{l+1}[n]\right)^{2}+y_{l+1}[n+1]-y_{l+1}[n]^{2} \leq\left(\frac{V T}{N}\right)^{2}, n=1,2, \ldots, N-1
\end{aligned}
$$

The overall algorithm which contains the solving process of two sub-problems can be given by bellow algorithm:

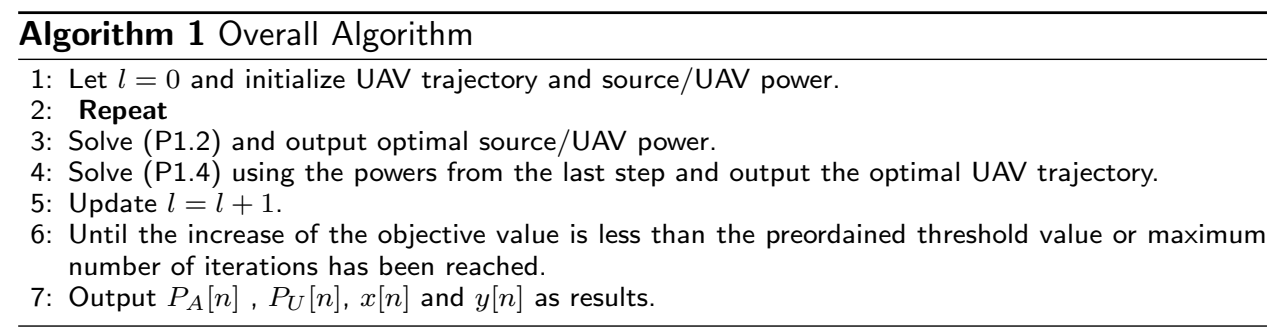




\section{Simulation Results and Discussion}

This section provides simulation results to verify the performance of the proposed algorithm. We assume the scenario that the distance between transmitter and receiver is $\mathrm{L}=2000$ meters. The altitude of $\mathrm{UAV}$ is constant and equal to at 100 meters. The bandwidth of communication channel between source and destination is $20 \mathrm{MHz}$. The noise power spectral density is $-100 \mathrm{dBm} / \mathrm{Hz}$ and the value of $\alpha_{0}$ is assumed $30 \mathrm{~dB}$. The maximum speed of UAV is $60 \mathrm{~m} / \mathrm{s}$. Another assumption is that the UAV flies from $(0,0,100)$ to $(2000,0,100)$ in 100 seconds. The maximum average transmission power at source and UAV are the same and equal to $10 \mathrm{dBm}$. For the first scenario with fixed trajectory, we assume directional trajectory from $(0,0,100)$ to $(2000,0,100)$ with a constant speed of $20 \mathrm{~m} / \mathrm{s}$. Fig. 2 is the output of power allocation with fixed trajectory. It presents that, when the UAV travels close to the source, it should transmit data with much more power because the link distance to destination is more. In this case, the transmit power of A is less. In other words, the transmit power of A increases as the transmit power of UAV decreases while traveling from source to destination. As we see in Fig. 2 in the middle of the trajectory at time $50 \mathrm{~s}$, the power of source and UAV is equal to $10 \mathrm{Mw}$ because of equal link distance.

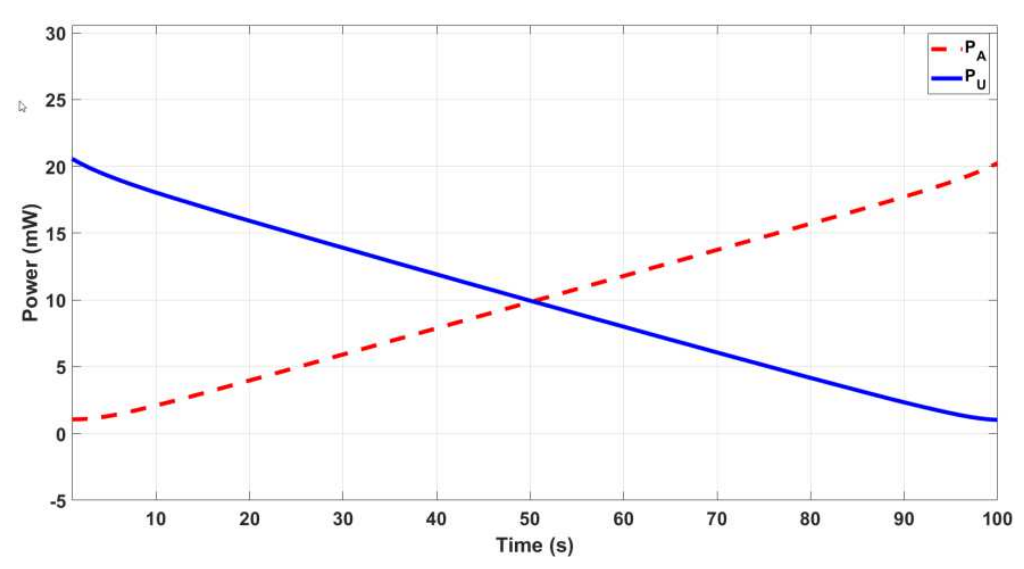

Figure 2 Power allocation with fixed trajectory

Fig. 3 shows the achievable signal to noise ratio and its equivalent information rate for the optimized power with fixed trajectory situation. The optimized information rate is about $3.97 \mathrm{bits} / \mathrm{s} / \mathrm{Hz}$.

In Fig.4 we show the value of our objective function according to iteration numbers to verify that the maximization procedure of the objective value is satisfied. As it is shown the value of objective function goes from 3.927 to 3.97 which is the optimized information rate.

In the second phase, we check out the case that the power of A and destination are fixed and equal to $\bar{P}=10 \mathrm{~mW}$ in the whole time of flying. Fig.5 shows the optimized $\mathrm{x}$ axis of the trajectory in 10 iterations. The UAV flies with its maximum speed to a place near the middle of the trajectory and hovers there for the longest time because the maximum information rate can be achieved there. The convergence of the output can be seen obviously. Both $\mathrm{x}$ and $\mathrm{y}$ axis of UAV for the last iteration 


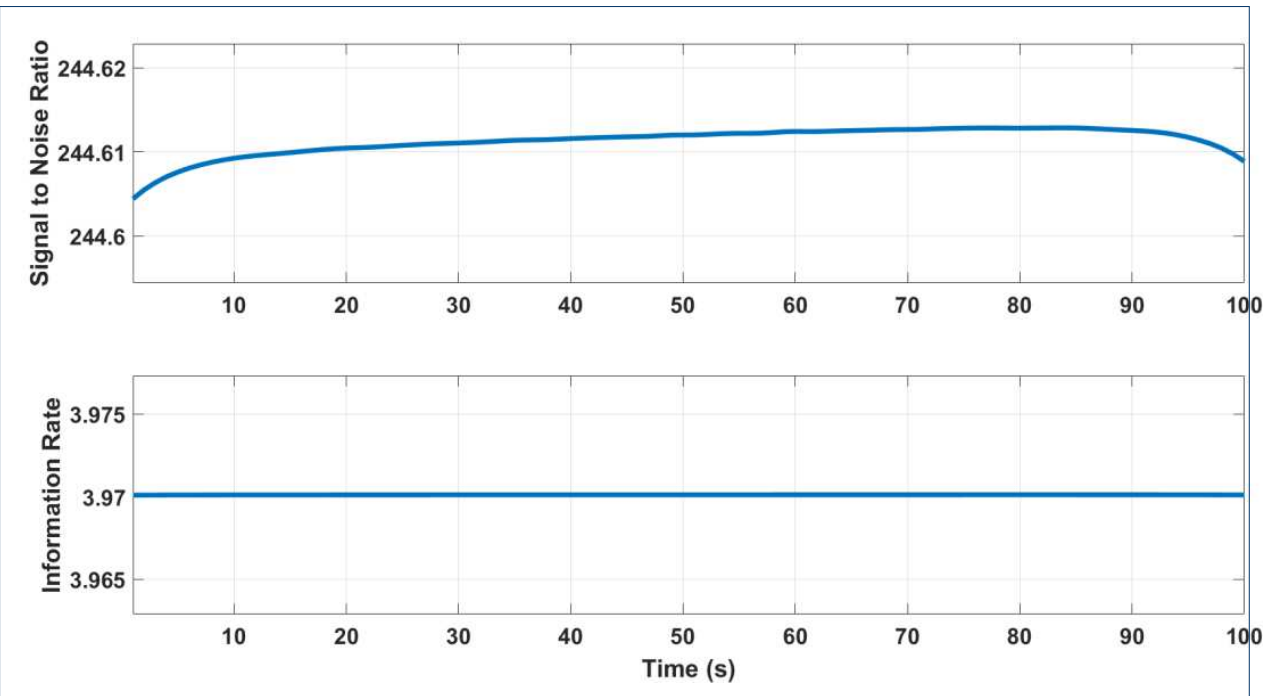

Figure 3 Optimized SNR and information rate for power allocation with fixed trajectory

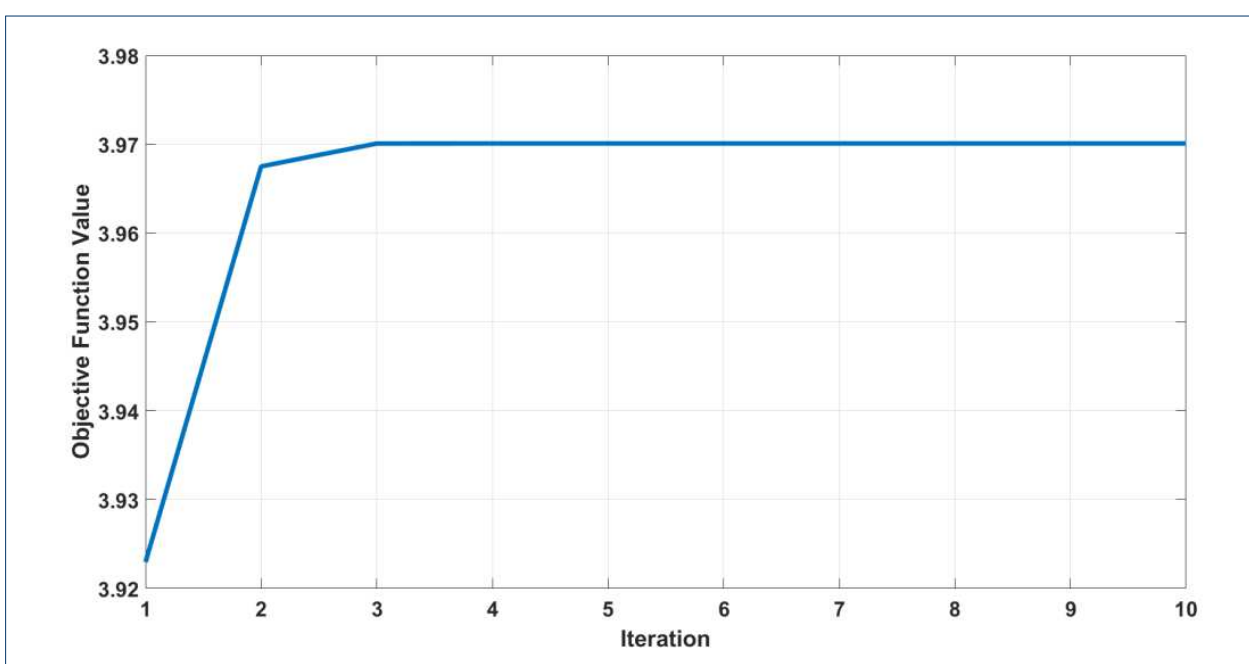

Figure 4 Objective function value versus iteration number

are plotted in Fig.6. As we see the optimal y axis is equal to zero. The reason is that it is favorable that link distance reaches minimum and lower power consumption we have.

In the following figures the optimal information rate by the second scenario and the value of objective function for the optimization algorithm which is the lower bound of information rate, are plotted. The value of objective function $R_{l b, l+1}[n]$ is equal to 3.485 which is lower than its equivalent rate in Fig.8. In Fig.8, The information rate increases while UAV flying from $(0,0,0)$ and gets fixed, as the location of UAV is fixed in Fig. 6 from 17s to 83s.

In the above figures, only one parameter has been optimized. In this part, the results of jointly power and trajectory optimization are presented. In Fig.9, the optimized trajectory with $\mathrm{x}$ and $\mathrm{y}$ axis is plotted. Like the previous part, the optimal $\mathrm{y}$ value is equal to zero due to minimizing link distanc. 


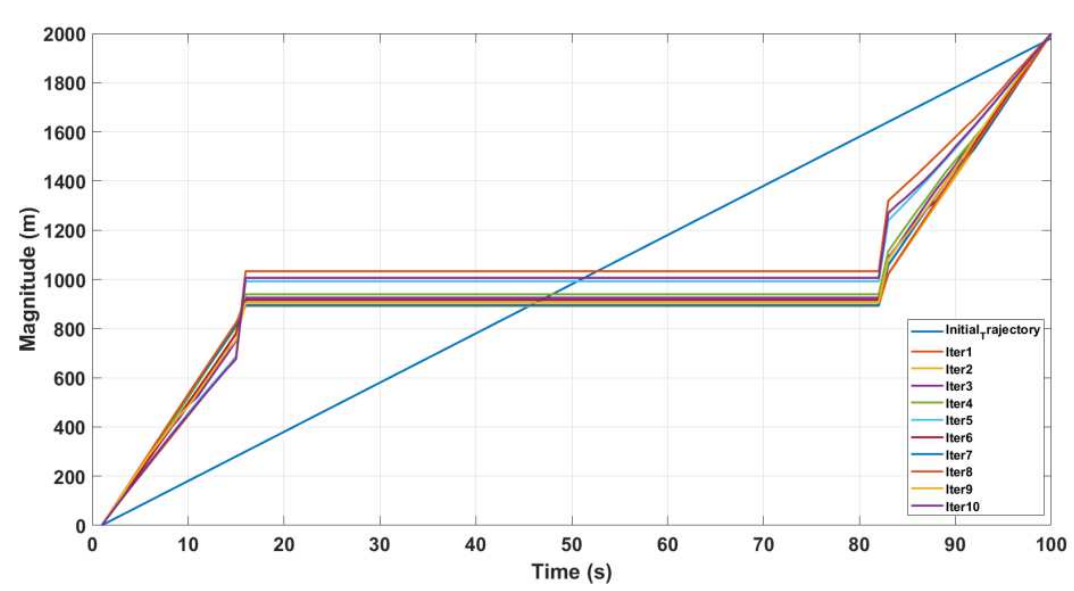

Figure $5 \mathrm{X}$ axis of optimized trajectory with fixed power allocation

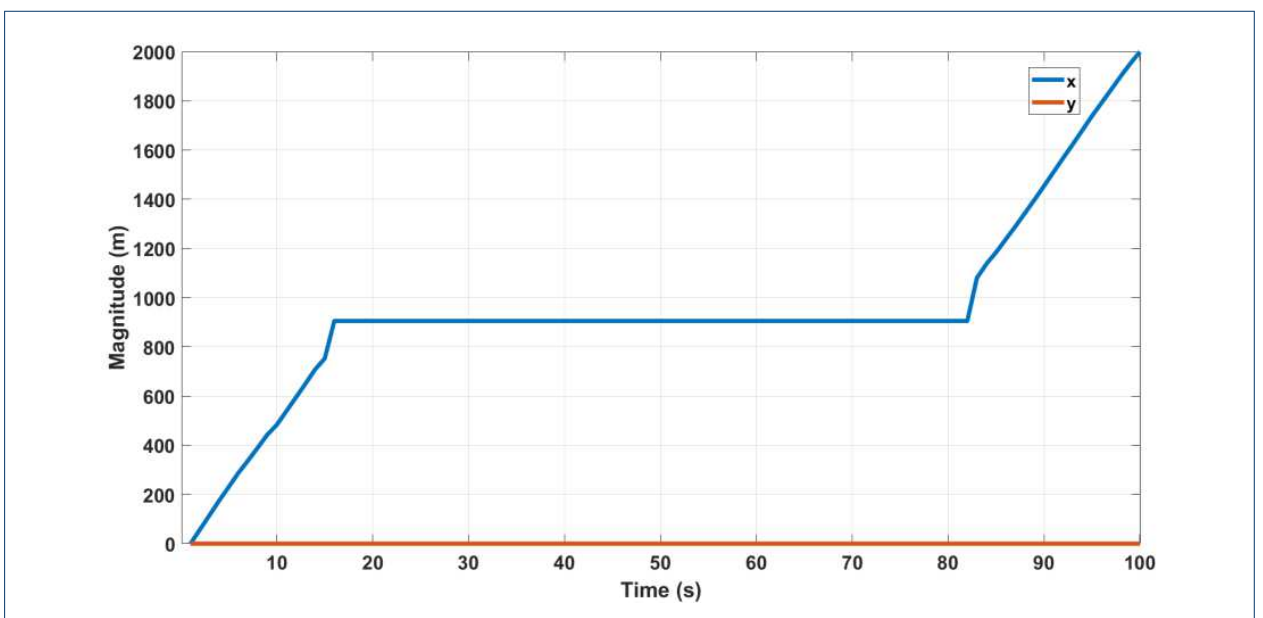

Figure $6 \mathrm{X}$ and $\mathrm{Y}$ axis of optimized trajectory with fixed power allocation

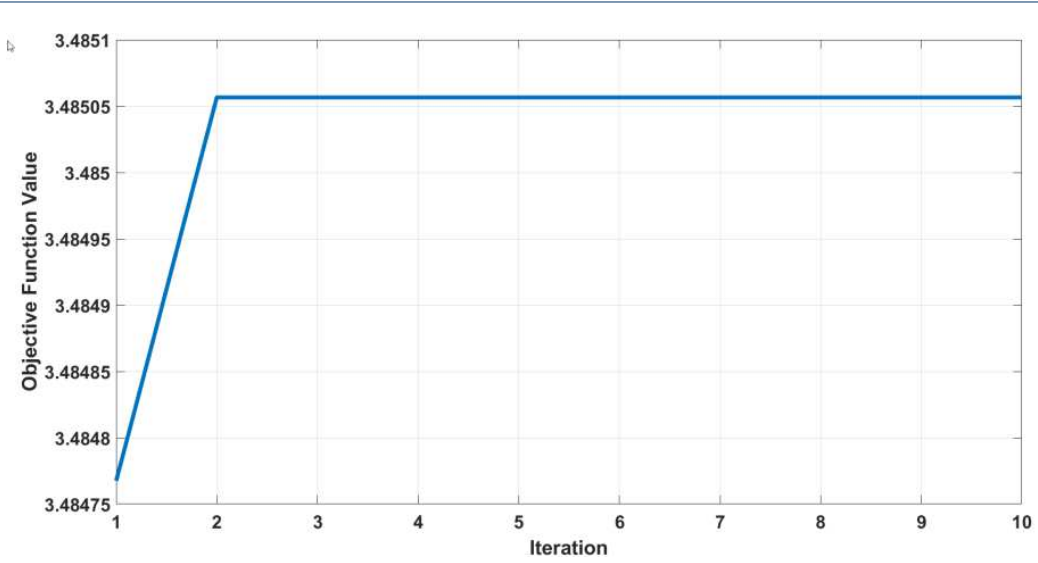

Figure 7 Objective function value versus iteration number

The UAV hovers for a long-time horizon from $17 \mathrm{~s}$ to $83 \mathrm{~s}$ in the position of 900 


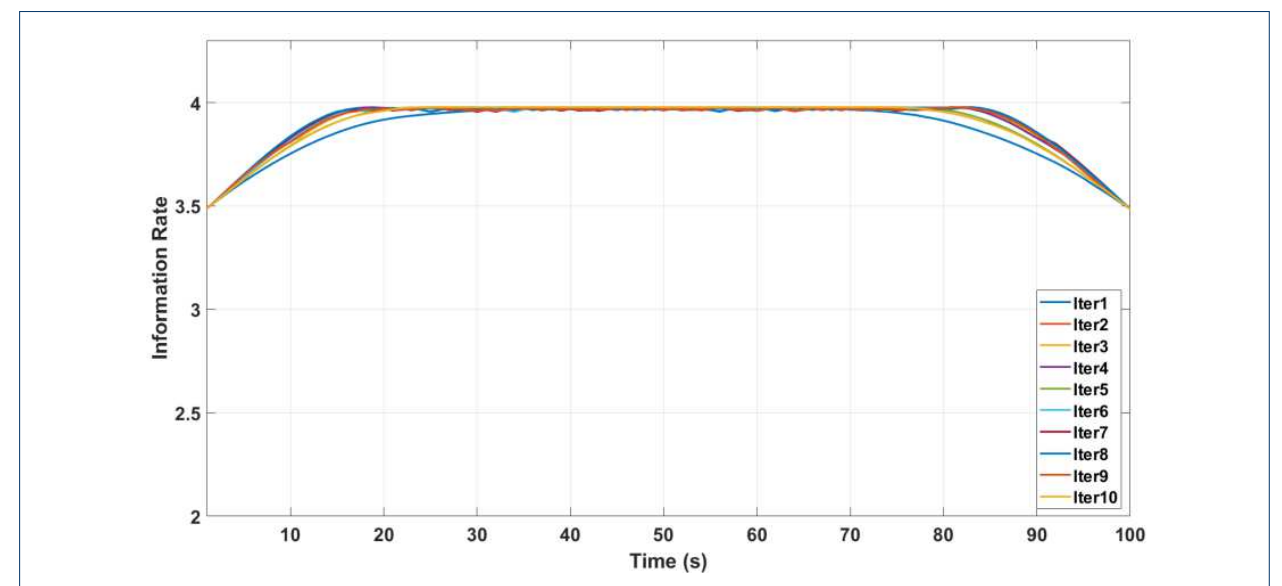

Figure 8 Optimal information rate for the trajectory optimization with fixed power allocation

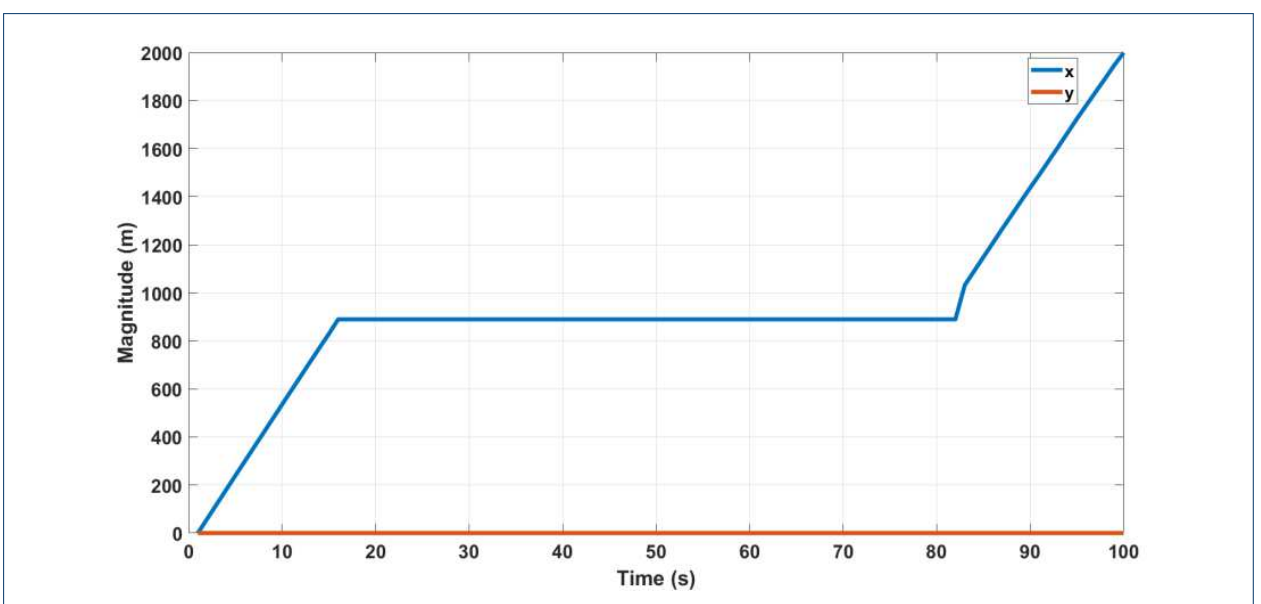

Figure 9 Optimized trajectory by jointly optimizing power and trajectory

meters. According to the expression for calculating information rate in (7), the information rate reaches maximum in this place with pre-determined $\bar{P}, \beta_{0}$ and $\sigma^{2}$. With this optimized trajectory, the power allocation scheme is like Fig.10.

It is explainable that for the time that UAV hovers in the middle of its trajectory, the power of source node $\mathrm{A}$ and UAV are almost equal. Before this time period, the UAV transmit with more power because it is close to A and its distance to B is more. From 83 to 100 s the transmission power of UAV decreases to $10 \mathrm{dBm}$ and the transmission power of A increases to $10 \mathrm{dBm}$ due to different link distances.

\section{Conclusion}

In this paper, a UAV-based relaying system, benefiting the UAV's mobility is studied. The minimum information rate of considered wireless network is maximized via optimizing both the source/relay power allocation and relay trajectory. To this end, we propose two iterative algorithms for fixed trajectory and fixed power allocation scenarios and find the optimal solution for the lower bound of the maximum rate. According to the results of proposed methods, an overall algorithm is derived which jointly optimizes the power allocations and UAV trajectory 


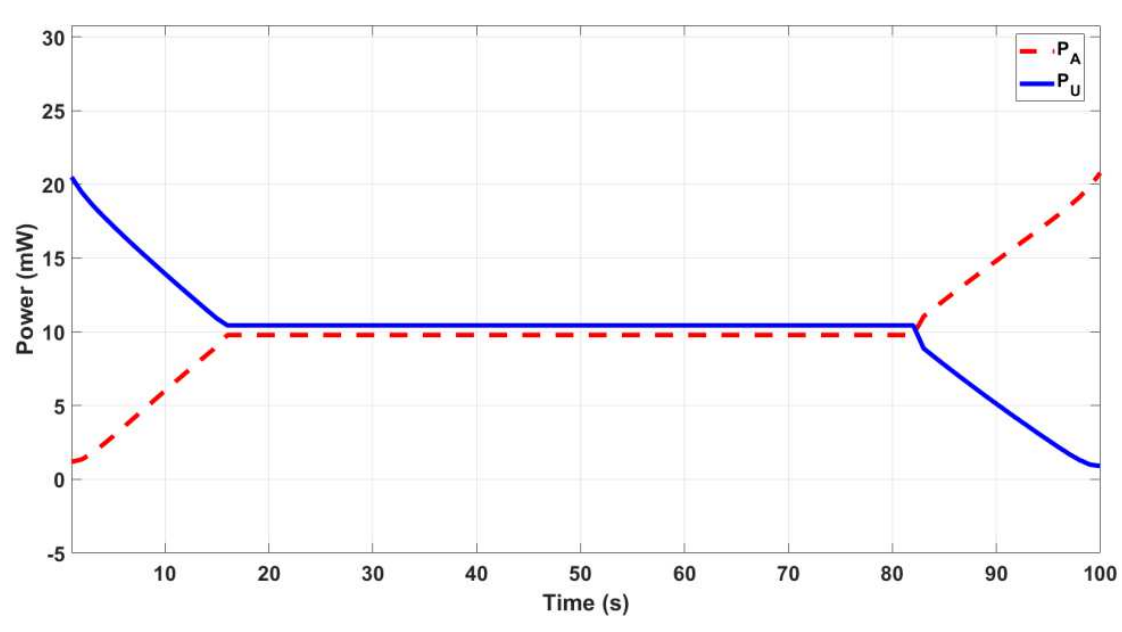

Figure 10 Power allocation by jointly optimizing power and trajectory

alternately. Simulation results demonstrate that a higher system rate can be achieved by considering mobile relay compared to static relay which is operational for future real wireless networks in temporary situations. For future work, we can intend interference scenarios and also NLOS channel caused by long buildings.

\section{Acknowledgements}

Not applicable.

Funding

Not applicable.

\section{Abbreviations}

UAV: unmanned aerial vehicle

5G: fifth-generation

IoT: internet of things

3GPP: 3rd generation partnership project

LOS: line-of-sight

GT: ground terminals

WPT: wireless power transfer

SWIPT: simultaneous wireless information and power transfer

D2D: device-to-device

SCO: successive convex optimization

AF: amplify-and-forward

DF: decode-and-forward

AWGN: additive white Gaussian noise

SNR: signal-to-noise ratio

Availability of data and materials

Not applicable

Competing interests

The authors declare that they have no competing interests.

Consent for publication

All authors have agree and given their consent for submission of this paper to Euraship Journal of Wireless Communications and Networking.

Authors' contributions

All authors contributed in designing the proposed schemes and also writing and reviewing the manuscript. They approved the final manuscript.

Authors' information

Sedighe Nasrollahi received the B.Sc. degree in communication engineering from Azad University of Mashhad, Mashhad, Iran in 2014 and the M.Sc. degree in communications engineering from the Shahrood University of 
Technology, Shahrood, Iran in 2018. Her current research interests include information theory, channel and source coding, cloud storage and information theoric learning.

Seyed Masoud Mirrezaei received his B.Sc. degree in Communication Engineering from K. N. Toosi University of Technology in Sept. 2004. He received his M.Sc. and Ph.D. degrees in Electrical Engineering from Amirkabir University of Technology, Tehran, Iran, in 2007 and 2013, respectively. He has been as a Ph.D. student and researcher in "Mobile and Wireless Networks Research Laboratory" in Amirkabir University of Technology under the supervision of Prof. Karim Faez. Also, he was a visiting research student in the Signal Design and Analysis Laboratory (SDAL) at Queen's University, Kingston, Canada under the supervision of Prof. Shahram Yousefi from March 2011 to February 2012. He joined the Electrical Engineering Department of Shahrood University of Technology from 2013 up to now. His research interests lie in communications, cloud systems, big data, networks, information theory, signal processing, channel coding and network coding.

\section{Author details}

Faculty of Electrical Engineering, Shahrood University of Technology, Shahrood, Iran.

References

1. Zhao, Y., Adve, R., Lim, T.J.: Improving amplify-and-forward relay networks: optimal power allocation versus selection. In: 2006 leee International Symposium on Information Theory, pp. 1234-1238 (2006)

2. Liu, X., Li, Z., Zhao, N., Meng, W., Gui, G., Chen, Y., Adachi, F.: Transceiver design and multihop d2d for uav iot coverage in disasters. IEEE Internet of Things Journal 6(8), 1803-1815 (2018)

3. Mozaffari, M., Saad, W., Bennis, M., Debbah, M.: Unmanned aerial vehicle with underlaid device-to-device communications: Performance and tradeoffs. IEEE Transactions on Wireless Communications 15(6), 3949-3963 (2016)

4. Matolak, D.W., Sun, R.: Air-ground channel characterization for unmanned aircraft systems-part iii: The suburban and near-urban environments. IEEE Transactions on Vehicular Technology 66(8), 6607-6618 (2017)

5. 36.777, G.T.: Study on Enhanced LTE Support for Aerial Vehicles, https://www.3gpp.org/ftp/Specs/archive/36 series/36.777/.

6. Yang, Z., Pan, C., Shikh-Bahaei, M., Xu, W., Chen, M., Elkashlan, M., Nallanathan, A.: Joint altitude, beamwidth, location, and bandwidth optimization for uav-enabled communications. IEEE Communications Letters 22(8), 1716-1719 (2018)

7. Chen, Y., Feng, W., Zheng, G.: Optimum placement of uav as relays. IEEE Communications Letters 22(2), 248-251 (2017)

8. Zeng, Y., Zhang, R., Lim, T.J.: Wireless communications with unmanned aerial vehicles: Opportunities and challenges. IEEE Communications Magazine 54(5), 36-42 (2016)

9. Yang, D., Wu, Q., Zeng, Y., Zhang, R.: Energy tradeoff in ground-to-uav communication via trajectory design. IEEE Transactions on Vehicular Technology 67(7), 6721-6726 (2018)

10. Xu, J., Zeng, Y., Zhang, R.: Uav-enabled multiuser wireless power transfer: Trajectory design and energy optimization. In: 2017 23rd Asia-Pacific Conference on Communications (APCC), pp. 1-6 (2017)

11. Yin, S., Zhao, Y., Li, L., Yu, F.R.: Uav-assisted cooperative communications with time-sharing information and power transfer. IEEE Transactions on Vehicular Technology 69(2), 1554-1567 (2019)

12. Zeng, Y., Zhang, R.: Energy-efficient uav communication with trajectory optimization. IEEE Transactions on Wireless Communications 16(6), 3747-3760 (2017)

13. Zeng, Y., Xu, J., Zhang, R.: Energy minimization for wireless communication with rotary-wing uav. IEEE Transactions on Wireless Communications 18(4), 2329-2345 (2019)

14. Dhillon, H.S., Huang, H., Viswanathan, H.: Wide-area wireless communication challenges for the internet of things. IEEE Communications Magazine 55(2), 168-174 (2017)

15. Liu, X., Gui, G., Zhao, N., Meng, W., Li, Z., Chen, Y., Adachi, F.: Uav coverage for downlink in disasters: Precoding and multi-hop d2d. In: 2018 IEEE/CIC International Conference on Communications in China (ICCC), pp. 341-346 (2018)

16. Liu, X., Li, Z., Zhao, N., Meng, W., Gui, G., Chen, Y., Adachi, F.: Transceiver design and multihop d2d for uav iot coverage in disasters. IEEE Internet of Things Journal 6(2), 1803-1815 (2018)

17. Na, Z., Mao, B., Shi, J., Wang, J., Gao, Z., Xiong, M.: Joint trajectory and power optimization for uav-relay-assisted internet of things in emergency. Physical Communication 41 (2020)

18. Mozaffari, M., Saad, W., Bennis, M., Debbah, M.: Unmanned aerial vehicle with underlaid device-to-device communications: Performance and tradeoffs. IEEE Transactions on Wireless Communications 15(6), 3949-3963 (2016)

19. Zeng, Q., Zhong, B., Sun, J., Liu, C., Wang, M.: Full-duplex relay aided device-to-device communication networks under the coverage of unmanned aerial vehicle base station. In: 2018 24th Asia-Pacific Conference on Communications (APCC), pp. 196-200 (2018)

20. Wang, H., Wang, J., Ding, G., Wang, L., Tsiftsis, T.A., Sharma, P.K.: Resource allocation for energy harvesting-powered $\mathrm{d} 2 \mathrm{~d}$ communication underlaying uav-assisted networks. IEEE Transactions on Green Communications and Networking 2(1), 14-24 (2017)

21. Wang, Q., Chen, Z., Mei, W., Fang, J.: Improving physical layer security using uav-enabled mobile relaying. IEEE Wireless Communications Letters 6(3), 310-313 (2017)

22. Cui, M., Zhang, G., Wu, Q., Ng, D.W.K.: Robust trajectory and transmit power design for secure uav communications. IEEE Transactions on Vehicular Technology 67(9), 9042-9046 (2018)

23. Mah, M.-C., Lim, H.-S., Tan, A.W.-C.: Secrecy improvement via joint optimization of uav relay flight path and transmit power. Vehicular Communications 23 (2020)

24. Zhou, X., Wu, Q., Yan, S., Shu, F., Li, J.: Uav-enabled secure communications: Joint trajectory and transmit power optimization. IEEE Transactions on Vehicular Technology 68(4), 4069-4073 (2019)

25. Zeng, Y., Zhang, R., Lim, T.J.: Throughput maximization for uav-enabled mobile relaying systems. IEEE Transactions on Communications 64(12), 4983-4996 (2016) 
26. Jiang, X., Wu, Z., Yin, Z., Yang, Z.: Joint power and trajectory design for uav-relayed wireless systems. IEEE Wireless Communications Letters 8(3), 697-700 (2018)

27. Wu, Q., Zeng, Y., Zhang, R.: Joint trajectory and communication design for multi-uav enabled wireless networks. IEEE Transactions on Wireless Communications 17(3), 2109-2121 (2018)

28. Fan, J., Cui, M., Zhang, G., Chen, Y.: Throughput improvement for multi-hop uav relaying. IEEE Access 7 , 147732-147742 (2019)

29. Cao, D., Yang, W., Li, N.: Throughput enhancement of buffer-aided multi-hop uav relaying wireless networks. AEU-International Journal of Electronics and Communications 131 (2021)

30. Li, Y., Wang, W., Gao, H., Wu, Y., Su, M., Wang, J., Liu, Y.: Air-to-ground 3d channel modeling for uav based on gauss-markov mobile model. AEU-International Journal of Electronics and Communications 114 (2020)

31. BoydandL, S.: Vandenberghe, Convex Optimization. cambridge university press (2004)

Figures

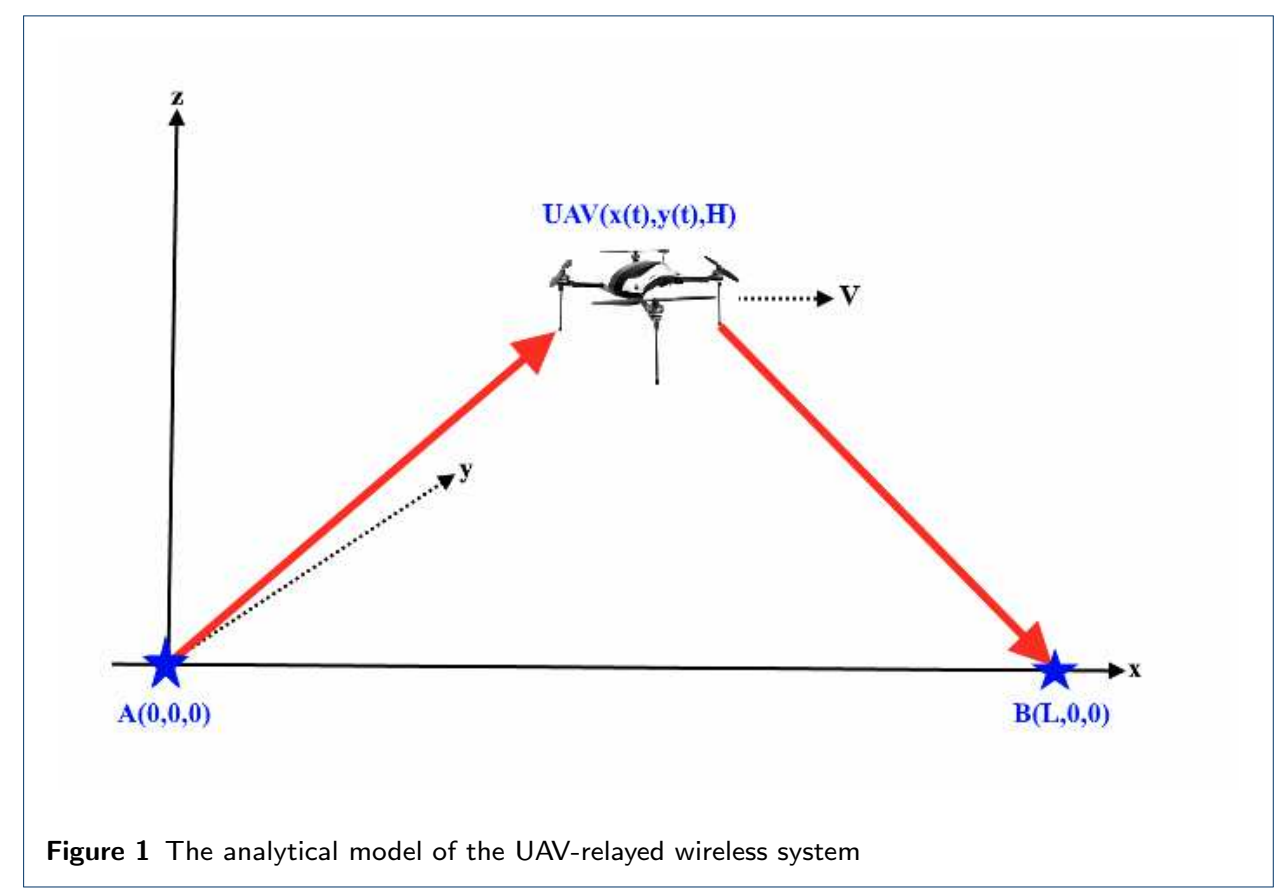




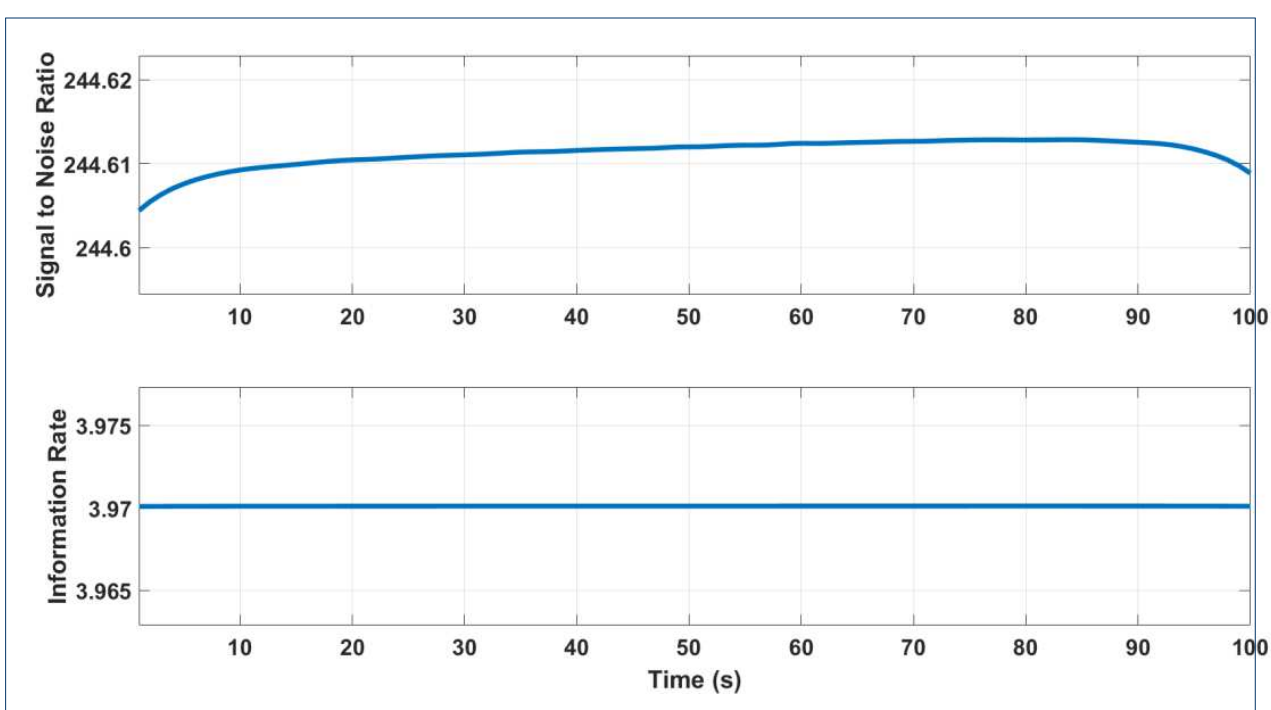

Figure 3 Optimized SNR and information rate for power allocation with fixed trajectory

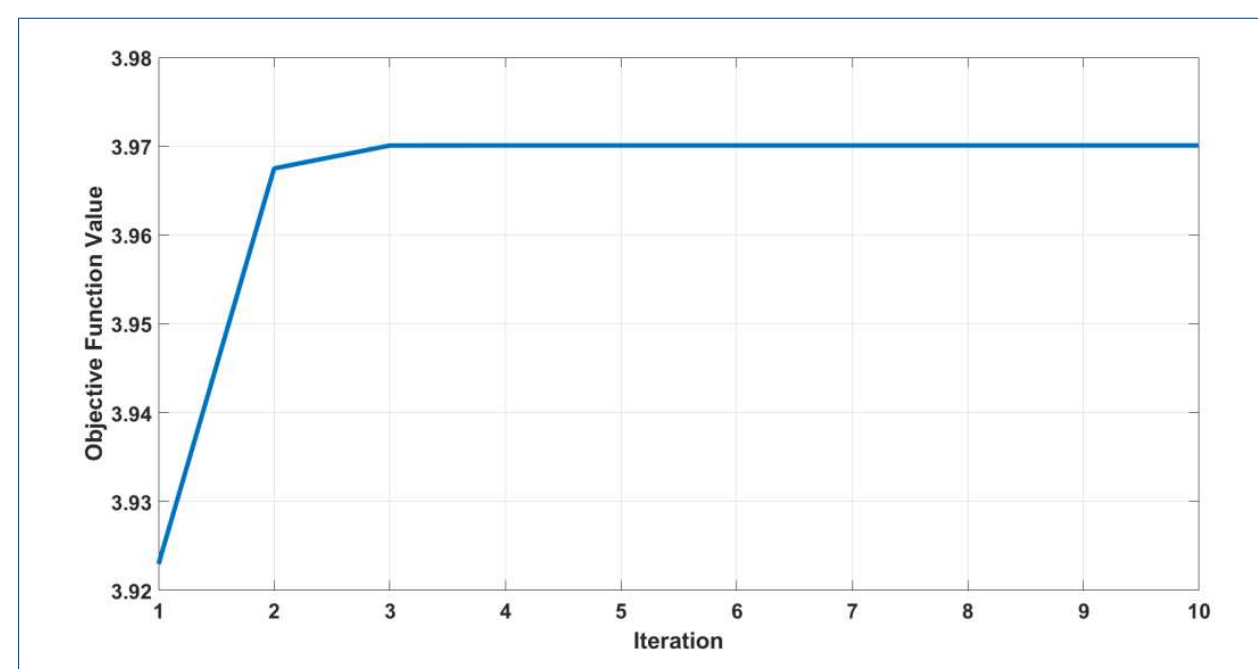

Figure 4 Objective function value versus iteration number

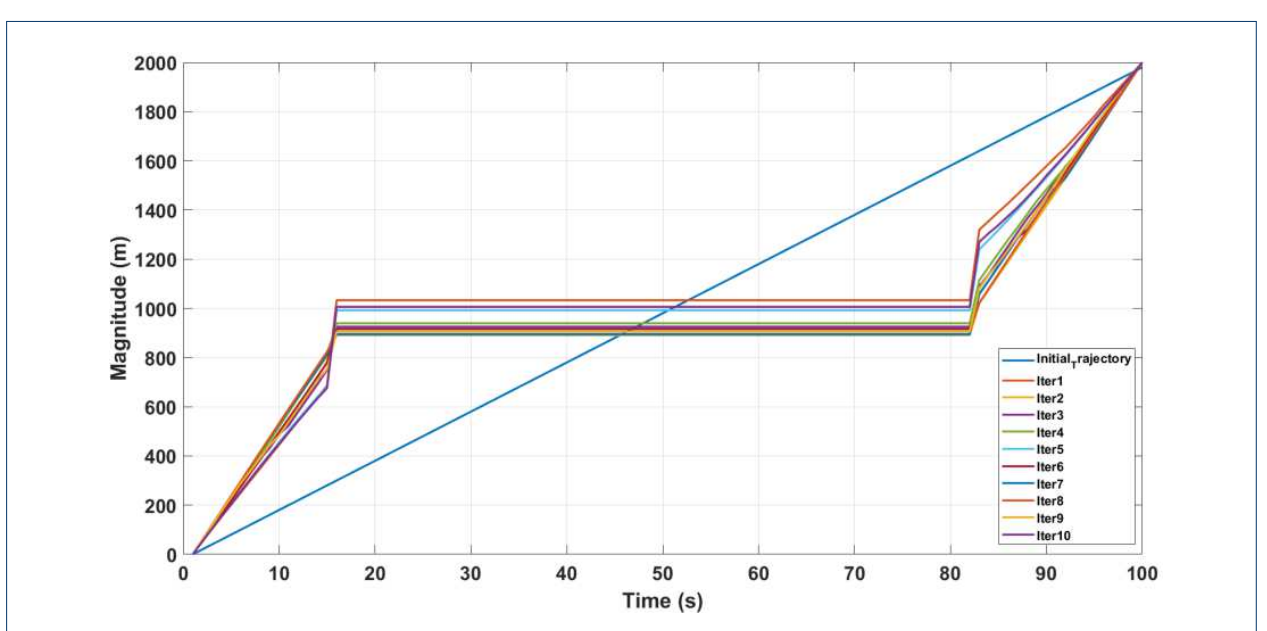

Figure $5 \mathrm{X}$ axis of optimized trajectory with fixed power allocation 
